\title{
Deberes escolares y rendimiento académico en Educación Primaria
}

\author{
Antonio Valle ${ }^{*}$, Irene Pan ${ }^{1}$, José C. Núñez ${ }^{2}$, Pedro Rosário ${ }^{3}$, Susana Rodríguez ${ }^{1}$ y Bibiana Regueiro ${ }^{1}$ \\ 1 Departamento de Psicología Evolutiva y de la Educación. Universidad de A Coruña (España) \\ 2 Departamento de Psicología. Universidad de Oviedo (España) \\ 3 Departamento de Psicologia. Universidad de Minho (Portugal)
}

\begin{abstract}
Resumen: En este artículo se analiza la relación entre la realización de deberes escolares y el rendimiento académico en una muestra de estudiantes de los tres últimos cursos de Educación Primaria. Las variables vinculadas con la implicación de los alumnos en los deberes escolares fueron el número de deberes realizados, el tiempo dedicado a los deberes y el aprovechamiento del tiempo dedicado a los deberes; mientras que el rendimiento académico fue estimado en base a las notas en matemáticas y lengua extranjera (inglés). Se ha tenido en cuenta el posible efecto del curso y del género a la hora de calcular el nivel predictivo de las variables relativas a los deberes sobre el rendimiento académico. Los resultados obtenidos indican que la cantidad de deberes realizados de los prescritos y el aprovechamiento del tiempo predicen positiva y significativamente el rendimiento académico en ambas asignaturas, mientras que la cantidad de tiempo dedicado a la realización de los deberes no es relevante. Finalmente, mientras que el género no resultó significativo en la predicción del rendimiento académico, el curso sí predijo negativamente el rendimiento en matemáticas: a medida que se asciende de curso el rendimiento en matemáticas tiende a disminuir.

Palabras clave: Deberes escolares; implicación en los deberes escolares; rendimiento académico; educación primaria.
\end{abstract}

\section{Introducción}

Los deberes escolares constituyen una de las actividades educativas que mayor controversia ha generado en los últimos años en diferentes sectores educativos. Dado que no existen unos criterios claros respecto a cómo, cuándo y cuántos deben ser prescritos, la polémica está lejos de tener una respuesta precisa sobre el grado de utilidad y de sus efectos sobre el aprendizaje de los estudiantes. A esto hay que añadir que la investigación sobre los deberes escolares tampoco ofrece una única respuesta a los múltiples interrogantes que surgen a la hora de recomendar su uso y, sobre todo, hay una ausencia de resultados coherentes que ofrezcan con claridad cuál es el camino a seguir en el futuro (Dettmers, Trautwein, y Lüdtke, 2009; Trautwein y Köller, 2003; Trautwein, Köller, Schmitz, y Baumert, 2002).

Uno de los principales propósitos de la asignación de deberes escolares es proporcionar a los estudiantes una oportunidad para practicar y revisar el material de aprendizaje que, previamente, se ha presentado en el aula (Becker y Epstein, 1982). Por eso, algunos autores (Cooper, 2001; Cosden, Morrison, Albanese y Macias, 2001) consideran los deberes escolares como una parte más del trabajo académico del alumnado, que se asignan para realizar fuera del periodo habitual de clases con el fin de extender y ampliar la práctica de habilidades académicas. Además, para muchos expertos, la reali-

* Dirección para correspondencia [Correspondence address]: Antonio Valle. Departamento de Psicología Evolutiva y de la Educación, Universidad de A Coruña, Campus de Elviña, s/n. 15071 A Coruña (España).E-mail: vallar@udc.es
Title: Homework and academic achievement in Primary Education. Abstract: This paper analyzes the relationship between homework behavior and academic achievement using a sample of students from the last three years of primary education. The variables associated with student involvement in homework were the amount of homework. completed, the time spent on homework, and bomework time optimization, while academic achievement was estimated based on the grades in mathematics and foreign language (english). The possible effect of the course and gender when calculating the level of predictive variables concerning the homework on academic achievement was analyzed. The results indicated that the amount of homework done and the optimization of the time dedicated at homework significantly and positively predicted academic performance in both subjects. However, the amount of time spent on homework was not relevant. Finally, while gender was not statistically significant in predicting academic achievement, the course itself was statistically significant: as the course advances the mathematics achievement tends to diminish.

Key words: Homework; involvement in homework; academic achievement; primary education.

zación de deberes contribuye a desarrollar unos buenos hábitos de estudio y promueven una mayor independencia y responsabilidad en el proceso de aprendizaje. Al mismo tiempo, los padres conocen más de cerca lo que aprenden sus hijos en la escuela y, de este modo, también se estrechan los vínculos familia-escuela (Epstein y Van Voorhis, 2001; Hill y Taylor, 2004).

No obstante, debido en cierta medida a la inconsistencia de los resultados de la investigación empírica, actualmente existe un gran debate sobre cómo el rendimiento académico de los estudiantes se ve afectado por la cantidad de deberes realizados y el tiempo dedicado a su realización. Con el presente estudio se pretende aportar datos a este debate centrando el análisis de dicha relación en los tres últimos cursos de Educación Primaria (incluyendo el efecto del curso), tomando en consideración el rendimiento en dos áreas curriculares muy diferentes (con el fin de analizar la consistencia de los resultados), contemplando la gestión del tiempo dedicado a la realización de los deberes como una posible variable crucial en dicha relación y, finalmente, considerando el posible efecto del género en dichos resultados.

\section{Tiempo dedicado a los deberes y rendimiento aca- démico}

La investigación pasada sobre la relación entre los deberes escolares y el rendimiento escolar ha estado muy centrada en cómo el tiempo empleado por los alumnos en la realización de las tareas asignadas incide en el rendimiento académico. Los resultados de la investigación son poco claros y, en algunos casos, contradictorios (Rosário et al., 2011). De 
hecho, el esfuerzo invertido en los deberes ha demostrado tener un impacto más positivo en el rendimiento académico que el tiempo en sí mismo dedicado a los deberes (Trautwein, Ludtke, Schnyder y Niggli, 2006). Además, varios estudios (De Jong, Westerhof y Creemers, 2000; Muhlenbruck, Cooper, Nye y Lindsay, 2000; Núñez et al., 2013; Trautwein, 2007) indican que los estudiantes que dedican más tiempo a los deberes no necesariamente obtienen mejores resultados que sus compañeros. En todo caso, la mayoría de los trabajos sólo encuentran una débil relación entre el tiempo dedicado a los deberes escolares y el rendimiento académico de los estudiantes (Senechal y LeFevre, 2002). Incluso, esta relación varía según la edad de los estudiantes, encontrándose una relación que va de modesta a débil en los cursos altos (Cooper y Valentine, 2001) y no habiendo relación en el caso de los estudiantes más jóvenes (Cooper, Lindsay, Nye y Greathouse, 1998).

Una posible explicación de la escasa relación entre tiempo dedicado a los deberes y el logro académico es la motivación de los estudiantes hacia los deberes (Trautwein et al., 2006). La investigación sugiere que el tipo de motivación de los estudiantes ante una tarea se relaciona con la calidad de su implicación (Ryan y Deci, 2000). Aunque no hay demasiada investigación sobre el tema, existen indicios de que muchos estudiantes participan en los deberes escolares no por el interés o entusiasmo que les producen, sino más bien por otras razones como, por ejemplo, el sentido del deber, el deseo de agradar o, incluso, por la evitación de castigos $\mathrm{Xu}$ y Wu, 2013; Walker, Hoover-Dempsey, Whetselm y Green, 2004). Esto es claramente negativo porque la motivación extrínseca suele estar relacionada con bajos niveles de persistencia, escasos aprendizajes, bajo rendimiento académico y con un mayor riesgo de abandonar la escuela (Vallerand, Fortier y Guay, 1997), sobre todo en comparación con la motivación intrínseca, que ha sido asociada con una serie de resultados, tales como la persistencia, la creatividad, el rendimiento, las emociones positivas y el interés en la escuela (Bouffard, Boileau, y Vezeau, 2001).

\section{Cantidad de deberes realizados y rendimiento aca- démico}

Algunos investigadores han encontrado que los estudiantes que completan sus deberes escolares tienen unas mejores calificaciones académicas que aquellos que no los completan (Cooper, 1989; Cooper, Robinson y Patall, 2006; Cooper y Valentine, 2001; Epstein y Van Voorhis, 2001; Trautwein et al., 2002). La importancia de completar los deberes se incrementa a medida que los estudiantes avanzan en la escuela (Zimmerman y Kitsantas, 2005).

El efecto de los deberes sobre el rendimiento académico parece que es menos relevante en Educación Primaria que en Educación Secundaria (Cooper, Jackson, Nye y Lindsay, 2001). Una de las razones por las cuales la relación entre deberes y rendimiento es más débil en Primaria que en Secundaria puede estar en que la asignación de deberes que hacen los profesores en los cursos de Primaria suele tener como objetivo prioritario que los alumnos aprendan a gestionar mejor su tiempo de estudio mediante una mera revisión del material de clase, mientras que el profesorado de Secundaria asigna esos deberes para enriquecer y perfeccionar las lecciones impartidas en clase (Muhlenbruck et al., 2000). Por eso, no sólo los estudiantes de Secundaria que hacen los deberes mejoran su rendimiento académico, sino que también esto suele ir acompañado de mejoras en su autoeficacia (Zimmerman y Kitsantas, 2005), sobre todo si el profesorado realiza los ajustes y adaptaciones necesarias para aquellos estudiantes con peores y con mejores niveles de rendimiento académico.

\section{Género, deberes escolares y rendimiento académico}

Un amplio número de trabajos (Covington, 1998; Deslandes y Cloutier, 2002; Harris, Nixon, y Rudduck, 1993; Jackson, 2003) sugieren que las niñas manifiestan actitudes más positivas que los niños hacia los deberes escolares y dedican mayores esfuerzos a realizarlos. Así, Harris et al. (1993) encontraron que las niñas organizan mejor su tiempo en relación con los deberes escolares. También Younger y Warrington (1996) consideran que las niñas trabajan de un modo más constante y consistente a la hora de hacer los deberes, realizando un trabajo más detallado y coherentemente planificado y mostrando más esfuerzo y mejores actitudes ante las adversidades. En líneas generales, las niñas suelen emplear mejores estrategias a la hora de hacer los deberes (Xu, 2007), dedican más tiempo a realizar los deberes (Trautwein, 2007; Wagner, Schober y Spiel, 2007) y muestran actitudes más positivas hacia ellos (Rosário, Mourão, Núñez, González-Pienda y Valle, 2006). Además, las niñas se muestran menos propensas a asistir a clase sin hacer los deberes y suelen considerarlos menos aburridos que los niños $(\mathrm{Xu}$, 2006). Al mismo tiempo, dedican mayores esfuerzos en gestionar su espacio de trabajo, regular su proceso motivacional y controlar aquellas emociones negativas que pueden surgir durante la realización de los deberes escolares (Xu, 2010).

\section{Objetivos del estudio}

Con este trabajo se pretende aportar información sobre la relación entre los deberes escolares y el rendimiento académico en Matemáticas y Lengua Inglesa en una muestra de estudiantes de los tres últimos cursos de Educación Primaria. Puesto que, la relación encontrada en Educación Primaria entre realización de deberes y rendimiento académico es poco consistente de un estudio a otro (con media cercana a cero), en este trabajo se analizó dicha relación operativizando las variables relativas a los deberes escolares como (a) cantidad de deberes realizados de los prescritos, (b) tiempo dedicado a la realización de los deberes y (c) gestión del tiempo empleado (aprovechamiento del tiempo), y tomando como criterio dos áreas curriculares teóricamente diferentes (Matemáticas y Lengua Inglesa) con el fin de comprobar si 
los resultados pudieran ser dependientes del área curricular. Además, en los análisis se tienen en cuenta el efecto del curso (se incluyen los tres últimos cursos de Primaria: $4^{\circ}, 5^{\circ}$ y $\left.6^{\circ}\right)$ y el género.

\section{Método}

\section{Participantes}

En el estudio participaron 326 estudiantes pertenecientes a dos centros públicos de Educación Primaria de la provincia de A Coruña. El 49.7\% son niños y el 5.3\% son niñas. De edades comprendidas entre los 9 y los 12 años, 75 de ellos cursaban $4^{\circ}$ curso de Educación Primaria (37 alumnos y 38 alumnas), 150 cursaban $5^{\circ}$ curso de Educación Primaria (75 alumnos y 75 alumnas) y 101 cursaban $6^{\circ}$ curso de Educación Primaria (50 alumnos y 51 alumnas).

\section{Instrumentos y medidas}

Para medir las variables vinculadas con los deberes se utilizó la Encuesta sobre los Deberes Escolares (EDE), que es una escala que evalúa diferentes dimensiones relativas a la eficacia de los deberes para el aprendizaje y rendimiento académico de los alumnos. En este estudio sólo se utiliza la información sobre las siguientes variables: a) número de deberes que realizan habitualmente los alumnos de los prescritos por los profesores, b) tiempo dedicado a los deberes y c) aprovechamiento del tiempo dedicado a los deberes. La estimación del número de deberes realizados por los alumnos se obtuvo mediante las respuestas a un ítem relativo a la cantidad de deberes realizados habitualmente, utilizando para ello una escala tipo likert con cinco alternativas $(1=$ ninguno, $2=$ algunos, $3=$ la mitad, 4 = casi todos, $5=$ todos). Posteriormente, de cara a simplificar la información obtenida, se dicotomizó dicha variable con dos opciones de respuesta: 1) no hacen todos los deberes habitualmente y 2) sí hacen todos los deberes habitualmente.

En cuanto al tiempo diario dedicado a la realización de los deberes, los estudiantes respondieron a tres ítems (en general, en una semana típica, en un fin de semana típico) con la formulación general "¿Cuánto tiempo sueles dedicar a la realización de los deberes?", siendo las opciones de respuesta $1=$ menos de 30 minutos, $2=$ de 30 minutos a una hora, $3=$ de una hora a hora y media, $4=$ de hora y media a dos horas, 5 $=$ más de dos horas.

Finalmente, el aprovechamiento del tiempo dedicado a los deberes se evaluó a través de las respuestas a tres ítems (en general, en una semana típica, en un fin de semana típico) en los que se les pedía que indicaran el nivel de aprovechamiento del tiempo dedicado habitualmente a los deberes, utilizando para ello la siguiente escala: $1=$ lo desaprovecho totalmente (me distraigo constantemente con cualquier cosa), $2=10$ desaprovecho más de lo que debiera, $3=$ regular, $4=10$ aprovecho bastante, $5=$ lo aprovecho totalmente (me concentro y hasta terminar no pienso en otra cosa).
Por último, la evaluación del rendimiento académico se obtuvo mediante las calificaciones académicas finales obtenidas en Lengua Inglesa y en Matemáticas por los alumnos participantes en el estudio.

\section{Procedimiento}

Como paso previo a la recogida de datos, se solicitó el consentimiento del centro y de los padres de los alumnos para participar en el estudio. Posteriormente, se aplicaron las encuestas a los alumnos durante el horario escolar. Las calificaciones académicas de las dos asignaturas se recogieron al finalizar el curso académico.

\section{Análisis de datos}

Con el fin de dar respuesta a los objetivos del estudio, en un primero momento se realizaron análisis multivariados de la varianza (MANOVA) tomando como factores el género y el curso y como variables dependientes la cantidad de deberes realizados, el tiempo dedicado a la realización de los deberes y el aprovechamiento del tiempo dedicado a los deberes. Se analiza tanto el contraste multivariado como el efecto principal de los dos factores, y su interacción, sobre cada una de las tres variables dependientes. Para el estudio del tamaño del efecto se aplican los criterios del clásico trabajo de Cohen (1988), en el que un tamaño del efecto pequeño es definido como $\eta_{\mathrm{p}}{ }^{2}=.010$ (equivalente a $d=.20$ ), un tamaño del efecto medio se define como $\eta_{\mathrm{p}}{ }^{2}=.059$ (equivalente a $d$ $=.50$ ), $\mathrm{y}$ un tamaño del efecto grande se define como $\eta_{\mathrm{p}}{ }^{2}=$ .138 (equivalente a $d=.80$ ).

Este primer análisis nos informa sobre la conveniencia o no de controlar el efecto de las variables curso y género a la hora de estimar la relación entre el rendimiento académico en Lengua Inglesa y en Matemáticas y las tres variables relativas a los deberes escolares (cantidad, tiempo y aprovechamiento del tiempo). En un segundo momento, se llevan a cabo análisis de regresión jerárquica con el fin de conocer el poder predictivo de las variables vinculadas con los deberes sobre el rendimiento académico en Lengua Inglesa y en Matemáticas, controlando estadísticamente el efecto de las variables género y curso.

\section{Resultados}

\section{Estadísticos descriptivos}

En la Tabla 1 se aportan los coeficientes de correlación de Spearman así como las medias, desviaciones típicas, asimetría y curtosis de las variables incluidas en este estudio. Se han realizado los análisis de correlación de Spearman porque alguna de las variables no son continuas. Se analizó la normalidad de la distribución de las variables en base al criterio adoptado por Finney y DiStefano (2006), quienes indican valores máximos de 2 y 7 para asimetría y curtosis, respectivamente. En base a esto, se puede concluir que las variables incluidas en este estudio presentan distribuciones normales. 
Tabla 1. Medias, desviaciones típicas, asimetría, curtosis y matriz de correlaciones.

\begin{tabular}{|c|c|c|c|c|c|c|c|}
\hline & 1 & 2 & 3 & 4 & 5 & 6 & 7 \\
\hline 1. Rend. acad. Lengua Inglesa & 1.000 & & & & & & \\
\hline 2. Rend. acad. Matemáticas & $.726^{* *}$ & 1.000 & & & & & \\
\hline 3. Curso $^{a}$ & .012 & $-.132 *$ & 1.000 & & & & \\
\hline 4. Género ${ }^{b}$ & .063 & -.013 & .000 & 1.000 & & & \\
\hline 5. Numero de deberes escolares realizados ${ }^{c}$ & $.379 * *$ & $.356^{* *}$ & -.065 & $.138^{*}$ & 1.000 & & \\
\hline 6. Tiempo dedicado a los deberes escolares & .027 & -.057 & $.210 * *$ & .019 & $.126^{*}$ & 1.000 & \\
\hline 7. Aprovechamiento del tiempo dedicado a los deberes escolares & $.159 * *$ & $.198^{* *}$ & $-.143 * *$ & $.147^{*}$ & $.322 * *$ & .029 & 1.000 \\
\hline$M$ & 3.460 & 3.444 & 5.079 & 1.503 & 1.751 & 2.610 & 4.046 \\
\hline DT & 1.311 & 1.297 & 0.731 & 0.501 & 0.432 & 1.173 & 0.948 \\
\hline Asimetría & -0.403 & -0.427 & -0.125 & -0.012 & -1.170 & 0.474 & -1.246 \\
\hline Curtosis & -1.033 & -0.974 & -1.119 & -2.012 & -0.636 & -0.550 & 1.732 \\
\hline
\end{tabular}

${ }^{a}$ Curso $\left(4=4^{\circ}\right.$ curso de Primaria, $5=5^{\circ}$ curso de Primaria, $6=6^{\circ}$ curso de Primaria $)$

${ }^{\mathrm{b}}$ Género $(1=$ niño, 2 = niña $)$.

c Número de deberes realizados $(1=$ no hacen todos los deberes, $2=$ sí hacen todos los deberes $)$.

${ }^{* *} p<.01 ; *_{p}<.05$

Teniendo en cuenta las correlaciones, el rendimiento académico en Lengua Inglesa mantiene una relación estadísticamente significativa y positiva con el rendimiento en $\mathrm{Ma}$ temáticas $(r=.728 ; p<.001)$, con el número de deberes realizados $(r=.381 ; p<.001)$ y con el aprovechamiento del tiempo dedicado a los deberes $(r=.218 ; p<.001)$. Esto nos indica que cuantos más deberes de los prescritos se realizan y cuanto mayor es el aprovechamiento del tiempo dedicado a esos deberes, mayor será el rendimiento académico en Lengua Inglesa. Sin embargo, el curso y el género no parecen mostrar relaciones significativas con el rendimiento en esta asignatura. En cuanto al rendimiento en Matemáticas, las correlaciones son muy similares a las del rendimiento en Lengua Inglesa, excepto que a medida que se asciende de curso el rendimiento académico en esta asignatura disminuye $(r=$ $.138 ; p<.05)$.

Por otro lado, a medida que se asciende en el curso hay un aumento en el tiempo dedicado a los deberes $(r=.201 ; p$ $<.001)$ pero una disminución en el aprovechamiento del tiempo que se dedica a ellos $(r=-.125 ; p<.05)$. En cuanto al género, las niñas no sólo hacen más cantidad de deberes $(r=$ .138; $p<.05)$ sino que también aprovechan más el tiempo que les dedican a esos deberes $(r=.113, p<.05)$.

Por lo que se refiere a las tres variables vinculadas directamente con los deberes escolares, hay relaciones positivas y estadísticamente significativas entre la cantidad de deberes realizados, el tiempo dedicado a ellos y el aprovechamiento del tiempo.

\section{Efecto del curso y del género sobre los deberes es- colares}

Para el análisis de esta relación se ha llevado a cabo un MANOVA con dos variables independientes (curso y género) y tres variables dependientes (número de deberes realizados de los prescritos, tiempo dedicado a los deberes y aprovechamiento del tiempo dedicado a los deberes). En la Tabla 2 se aportan los estadísticos descriptivos relativos a este análisis.

Tabla 2. Estadísticos descriptivos (media, desviación típica) correspondientes a las variables "número de deberes", "tiempo dedicado a los deberes" y "aprovechamiento del tiempo dedicado a los deberes" en función del curso y del género.

\begin{tabular}{|c|c|c|c|c|c|c|}
\hline & \multicolumn{2}{|c|}{ Número de deberes realizados } & \multicolumn{2}{|c|}{ Tiempo dedicado a los deberes } & \multicolumn{2}{|c|}{ Aprovechamiento del tiempo dedicado a los deberes } \\
\hline & $M$ & $S D$ & $M$ & $S D$ & $M$ & $S D$ \\
\hline \multicolumn{7}{|c|}{$4^{\circ}$ Educación Primaria } \\
\hline Niños & 1.621 & 0.491 & 2.108 & 1.125 & 4.108 & 1.021 \\
\hline Niñas & 1.921 & 0.272 & 2.052 & 1.089 & 4.368 & 0.819 \\
\hline Total & 1.773 & 0.421 & 2.080 & 1.099 & 4.240 & 0.927 \\
\hline \multicolumn{7}{|c|}{$5^{\circ}$ Educación Primaria } \\
\hline Niños & 1.720 & 0.452 & 2.680 & 1.092 & 3.920 & 0.896 \\
\hline Niñas & 1.826 & 0.381 & 2.853 & 1.259 & 4.160 & 0.944 \\
\hline Total & 1.773 & 0.420 & 2.766 & 1.178 & 4.040 & 0.925 \\
\hline \multicolumn{7}{|c|}{$6^{\circ}$ Educación Primaria } \\
\hline Niños & 1.700 & 0.462 & 2.740 & 0.964 & 3.840 & 0.933 \\
\hline Niñas & 1.705 & 0.460 & 2.803 & 1.249 & 3.980 & 1.029 \\
\hline Total & 1.703 & 0.459 & 2.772 & 1.112 & 3.910 & 0.980 \\
\hline \multicolumn{7}{|l|}{ Muestra total } \\
\hline Niños & 1.691 & 0.463 & 2.567 & 1.085 & 3.938 & 0.937 \\
\hline Niñas & 1.811 & 0.392 & 2.652 & 1.256 & 4.152 & 0.950 \\
\hline Total & 1.751 & 0.432 & 2.610 & 1.173 & 4.046 & 0.948 \\
\hline
\end{tabular}


A nivel multivariado, los resultados obtenidos indican que tanto el efecto del curso $\left(\lambda_{\text {wilks }}=.919 ; F_{(6,636)}=4.547 ; p\right.$ $\left.<.001 ; \eta_{\mathrm{p}}^{2}=.041\right)$ como del género $\left(\lambda_{\text {Wilks }}=.972 ; F_{(3,318)}=\right.$ $\left.3.018 ; p<.05 ; \eta_{\mathrm{p}}^{2}=.028\right)$ son estadísticamente significativos, aunque el tamaño del efecto es pequeño en ambos casos Por otro lado, la interacción entre género y curso no es estadísticamente significativa $\left(\lambda_{\text {Wilks }}=.981 ; F_{(6,636)}=1.029\right.$; $\left.p=.055 ; \eta_{\mathrm{p}}^{2}=.010\right)$.

En cuanto al efecto del curso, los análisis univariados indican que existen diferencias estadísticamente significativas entre los cursos en el tiempo dedicado a los deberes $\left(F_{(2,320)}=\right.$ $\left.1.454 ; p<.001 ; \eta_{\mathrm{p}}^{2}=.061\right)$, incrementándose éste con el paso de $4^{\circ}$ a $6^{\circ}$ (ver Figura 1 ), siendo medio el tamaño del efecto. Sin embargo, no hay diferencias estadísticamente significativas entre los cursos en la cantidad de deberes realizados de los prescritos $\left(F_{(2,320)}=0.929 ; p=.396 ; \eta_{\mathrm{p}}^{2}=.006\right)$, ni tampoco en el aprovechamiento del tiempo dedicado a los mismos $\left(F_{(2,320)}=\right.$ 2.617; $\left.p=.075 ; \eta_{\mathrm{p}}^{2}=.016\right)$.

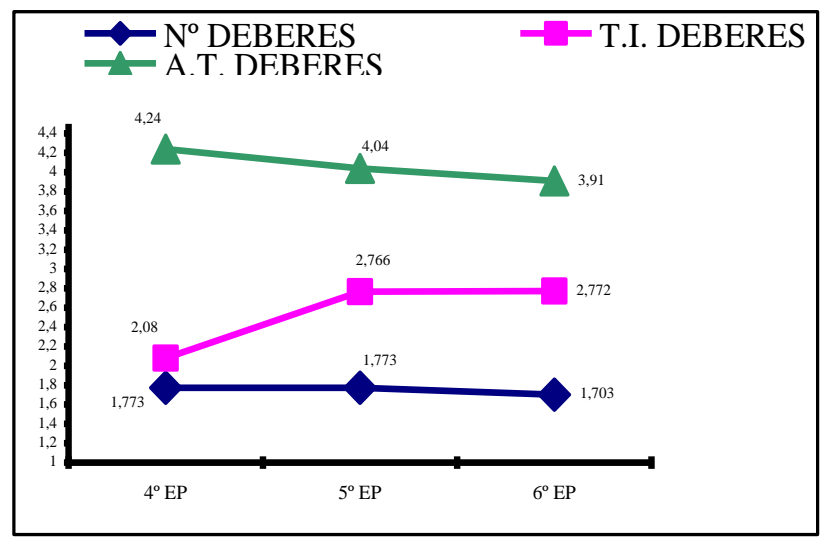

Figura 1. Representación gráfica de los niveles de las variables vinculadas con la implicación en los deberes escolares (número de deberes realizados $\mathrm{N}^{\circ}$ DEBERES-, tiempo dedicado a los deberes -T.I. DEBERES-, aprovechamiento del tiempo dedicado a los deberes -A.T. DEBERES) según el curso.

En relación al efecto del género, los análisis univariados muestran que hay diferencias estadísticamente significativas entre niños y niñas tanto en la cantidad de deberes realizados de los prescritos $\left(F_{(1,320)}=7.772 ; p<.05 ; \eta_{\mathrm{p}}^{2}=.024\right)$, como en el aprovechamiento del tiempo $\left(F_{(1,320)}=3.871 ; p<.05 ; \eta_{\mathrm{p}}{ }^{2}=.012\right)$, aunque el tamaño del efecto es pequeño en ambos casos. Por el contrario, no se encontraron diferencias estadísticamente significativas de género en el tiempo que dedican a bacer los deberes $\left(F_{(1,320)}=0.211 ; p=.646 ; \eta_{\mathrm{p}}^{2}=.001\right)$. En base a las medias de ambos grupos (ver Tabla 2 y Figura 2), se observó que las niñas realizan más cantidad de deberes de los prescritos (en $4^{\circ}$ y $5^{\circ}$ curso) y aprovechan mejor el tiempo dedicado a esos deberes que los niños.

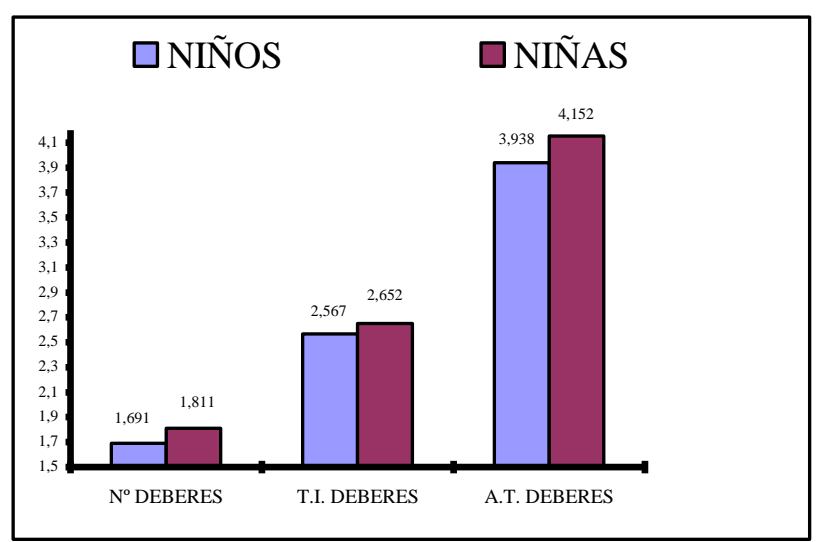

Figura 2. Representación gráfica de los niveles de las variables vinculadas con la implicación en los deberes escolares (número de deberes realizados No DEBERES-, tiempo dedicado a los deberes -T.I. DEBERES-, aprovechamiento del tiempo dedicado a los deberes -A.T. DEBERES) según el género.

Indicar, por último, que no se ha encontrado interacción estadísticamente significativa entre curso y género, ni en la cantidad de deberes realizados $\left(F_{(2,320)}=2.572 ; p=.078 ; \eta_{p}{ }^{2}=\right.$ .016), ni en el tiempo dedicado a los deberes $\left(F_{(2,320)}=0.257 ; p=\right.$ .773; $\left.\eta_{p}{ }^{2}=.002\right)$, ni tampoco en el aprovechamiento del tiempo dedicado a los deberes $\left(F_{(2,320)}=0.114 ; p=.892 ; \eta_{\mathrm{p}}^{2}=.001\right)$.

\section{Predicción del rendimiento académico en Lengua Inglesa y en Matemáticas}

Mediante el análisis de regresión jerárquica se pretende conocer el valor predictivo que tienen las tres variables vinculadas con los deberes escolares sobre el rendimiento académico en Lengua Inglesa y en Matemáticas. Teniendo en cuenta que las variables curso y género mantienen relación significativa con las variables vinculadas con los deberes escolares, se introdujeron como variables predictoras en la primera etapa de los análisis de regresión, con variables criterio Matemáticas y Lengua Inglesa. Con esto se trata de averiguar la cantidad de varianza que explican del rendimiento y así conocer, en una segunda etapa de los análisis de regresión, en qué medida las variables vinculadas con los deberes escolares predicen el rendimiento académico en Lengua Inglesa y en Matemáticas. Al realizar análisis de regresión por separado para Matemáticas y Lengua Inglesa es posible comprobar en qué medida los resultados son dependientes del área curricular examinada.

\section{Predicción del rendimiento en Lengua Inglesa}

Considerando la primera etapa del análisis de regresión jerárquica se observa que las variables curso y género sólo alcanzan a explicar, conjuntamente, el $0.5 \%$ de la varianza del rendimiento en esta asignatura, no alcanzando la significación estadística $\left(F_{(2,323)}=0.760 ; p=.468\right)$. Por tanto, ni género $(\beta=.068 ; t=1.227 ; p=.221)$ ni curso $(\beta=.007 ; t=$ 
$0.118 ; p=.906)$ son variables relevantes para explicar la variabilidad en el rendimiento en esta asignatura.

En la segunda etapa del análisis de regresión, además de las variables curso y género, se introducen en la ecuación de regresión las tres variables vinculadas con los deberes escolares (cantidad, tiempo y aprovechamiento del tiempo). Según se puede observar en la Tabla 3, de las tres variables, la cantidad de deberes realizados de los prescritos es la que más varianza explica del rendimiento en Lengua Inglesa $\left(F_{(3,322)}=18.393\right.$; $p<.001)$; un $14.1 \%$ de varianza explicada total $(\beta=.347 ; \mathrm{t}=$
6.355; $p<.001)$, lo que indica que tiene una capacidad predictiva relevante sobre el rendimiento académico en esta asignatura. Además de ésta, también es significativa la contribución del aprovechamiento del tiempo dedicado a la realización de los deberes $\left(F_{(4,321)}=14.905 ; p<.001\right)$, aportando un $1.1 \%$ de explicación de la variabilidad del rendimiento académico en esta asignatura $(\beta=.109 ; t=1.984 ; p<.05)$. Por el contrario, no resultó ser significativa la contribución de la variable cantidad de tiempo dedicado a la realización de los deberes $(\beta=$ $-.031 ; t=-0.586 ; p=.559)$.

Tabla 3. Varianza explicada $\left(R^{2}\right)$, coeficientes de regresión $(\beta)$ y estadístico y significación asociados $\left(t_{(\beta<)}\right)$ en la predicción del rendimiento académico en Lengua Inglesa y Matemáticas.

\begin{tabular}{lccc}
\hline & $R^{2}$ & $\beta$ & $t(p<)$ \\
\hline RENDIMIENTO EN LENGUA INGLESA & & & \\
Primera etapa (Modelo 1) & .005 & .007 & 0.118 \\
Curso & & .068 & 1.227 \\
Género & .152 & & \\
Segunda etapa (Modelo 2) & & .042 & 0.816 \\
Curso & & .008 & 0.151 \\
Género & .347 & $6.355^{* * *}$ \\
Número de deberes realizados & -.031 & -0.586 \\
Tiempo dedicado a los deberes & .109 & $1.984^{*}$ \\
Aprovechamiento del tiempo & & \\
RENDIMIENTO EN MATEMÁTICAS & & \\
Primera etapa (Modelo 1) & .019 & -.138 & $-2.505^{*}$ \\
Curso & & -.009 & -0.169 \\
Género & & \\
Segunda etapa (Modelo 2) & .148 & -.100 & $-1.947^{*}$ \\
Curso & & -.069 & -1.345 \\
Género & & .319 & $5.869^{* * *}$ \\
Número de deberes realizados & -.069 & -1.310 \\
Tiempo dedicado a los deberes & .142 & $2.605^{* *}$ \\
Aprovechamiento del tiempo & & &
\end{tabular}

\section{Predicción del rendimiento en Matemáticas}

Inicialmente, se observa que las variables curso y género explican conjuntamente el $1.9 \%$ de la varianza del rendimiento en Matemáticas. En este caso, aunque la cantidad de varianza explicada es baja, es estadísticamente significativa $\left(F_{(2,323)}=3.153 ; p<.05\right)$, quizás debido a que únicamente el curso tiene capacidad predictiva estadísticamente significativa $(\beta=-.138 ; t=-2.505 ; p<.05)$, y no el género $(\beta=-.009 ; t$ $=-0.169 ; p=.866)$. El signo negativo del coeficiente de regresión del curso está indicando que a medida que los alumnos avanzan de curso, su rendimiento en Matemáticas decrece.

En la segunda etapa del análisis de regresión, además de las variables curso y género, se introducen en la ecuación de regresión las tres variables vinculadas con los deberes escolares (cantidad, tiempo y aprovechamiento del tiempo). De nuevo, la variable con mayor poder predictivo del rendimiento, en este caso matemático, es el número de deberes realizados de los prescritos, siendo su contribución estadísticamente significativa $\left(F_{(3,322)}=18,617 ; p<.001 ; \beta=.319 ; t=5.869 ; p\right.$
$<.001$ ), aportando un $12.9 \%$ de varianza explicada (ver Tabla 3). Así como ocurriera con la predicción del rendimiento en Lengua Inglesa, el aprovechamiento del tiempo dedicado a los deberes también contribuye significativamente a la predicción del rendimiento en Matemáticas, aportando un $1.9 \%$ de varianza adicional $\left(F_{(4,321)}=15.911 ; p<.001\right)$, siendo el coeficiente beta estadísticamente significativo $(\beta=.142 ; t=$ 2.605; $p<.01)$. Y también al igual que en el caso de la Lengua Inglesa, en la predicción del rendimiento en Matemáticas, el tiempo dedicado a la realización de los deberes no se muestra una variable relevante. $(\beta=-.069 ; t=-1.310 ; p=.191)$.

\section{Discusión}

Con el presente estudio se pretendió aportar información sobre la relación entre algunas variables relativas a la conducta de los alumnos a la hora de realizar los deberes escolares y el rendimiento académico. Este estudio presenta algunas fortalezas que hacen, en algún modo, relevantes los resultados obtenidos. En primer lugar, pone su objetivo en los últimos cursos de Educación Primaria, etapa educativa en la que los resultados de los estudios previos son especialmente 
contradictorios y, sin embargo, es un momento evolutivo crucial por ser la transición a la etapa de Secundaria. En segundo lugar, se operativizan las variables relativas a los deberes escolares en cantidad de deberes realizados de los prescritos (y por tanto, ¿'en qué medida es importante realizar los deberes que se asignan para el rendimiento académico?), tiempo dedicado a su realización y aprovechamiento de ese tiempo (lo que permitirá interpretar mejor la relación entre tiempo utilizado en la realización de los deberes y rendimiento conseguido). En tercer lugar, se ha analizado dicha relación para dos asignaturas teóricamente muy diferentes, con el fin de comprobar si los resultados contradictorios podrían ser debidos al tipo de actividad curricular.

Los resultados de este trabajo ponen de manifiesto que cada una de las variables vinculadas con la implicación en los deberes escolares tiene efectos diferenciales en el rendimiento académico de los alumnos de los últimos cursos de Educación Primaria. Al mismo tiempo, tanto el curso como el género tienen efectos distintos sobre la implicación de los alumnos en los deberes escolares.

El número de deberes realizados y el aprovechamiento del tiempo predicen positiva y significativamente el rendimiento académico en Lengua Inglesa. Cuanto mayor número de deberes realizan los alumnos y cuanto más aprovechan el tiempo dedicado a esos deberes, mayor es el rendimiento académico en Lengua Inglesa. Sin embargo, ni el curso ni el género son predictores significativos del rendimiento en esta asignatura. En el caso del rendimiento en Matemáticas, el número de deberes realizados y el aprovechamiento del tiempo también predicen significativamente y en sentido positivo el rendimiento en esta asignatura. Aquí sí aparece el curso como un predictor significativo del rendimiento, aunque en sentido negativo, lo que indica que a medida que se asciende de curso, el rendimiento en Matemáticas tiende a disminuir.

Por tanto, en consonancia con otros estudios (Núñez et al., 2013; Trautwein, et al., 2002; Trautwein, Schnyder, Niggli, Neumann y Lüdtke, 2009), a mayor número de deberes realizados de los prescritos, mayor es el rendimiento académico obtenido por los alumnos. A esto hay que añadir que, además de esta variable, también resulta relevante el aprovechamiento del tiempo dedicado a la realización de los deberes. En definitiva, mucho más determinante que el tiempo que dedican los estudiantes a hacer los deberes, lo es el aprovechamiento que hacen de ese tiempo (Núñez et al., 2013). Por eso, aunque no se haya investigado directamente el efecto de esta variable, sí hay estudios que ponen de relieve el importante papel desempeñado por el esfuerzo dedicado a la realización de los deberes escolares. De hecho, un amplio número de investigaciones han encontrado que el esfuerzo invertido en realizar los deberes escolares predice positiva y significativamente el rendimiento académico (Trautwein, 2007; Trautwein et al., 2006; Trautwein et al., 2009; Zimmerman y Kitsantas, 2005). Por tanto, parece demostrado que el esfuerzo invertido en los deberes tiene un impacto más positivo en el rendimiento académico que el tiempo dedicado a los deberes (Trautwein et al., 2006).

Sin embargo, la mayoría de los trabajos sólo encuentran una débil relación entre el tiempo dedicado a los deberes escolares y el rendimiento académico de los estudiantes (Senechal y LeFevre, 2002). Todo parece indicar que el tiempo invertido por el alumno en los deberes escolares no es en sí mismo una garantía de su implicación y compromiso en la realización de las tareas asignadas (Rosário, Mourão, Núñez y Solano, 2008; Rosário et al., 2009; Trautwein et al., 2009). Es más, emplear un exceso de tiempo en la realización de las tareas puede significar, en ocasiones, una baja competencia cognitiva del estudiante e, incluso, dificultades para gestionar adecuadamente los recursos personales y ambientales relacionados con sus procesos de estudio y de trabajo individual.

En cuanto a la variable género, los resultados obtenidos indican que las niñas realizan mayor número de deberes escolares (en $4^{\circ}$ y $5^{\circ}$ curso) y aprovechan más el tiempo dedicado a esos deberes que los niños. Algunos de estos resultados coinciden con los aportados por otros estudios (Rosário et al., 2006; Xu, 2006, 2007, 2010; Wagner et al., 2007; Younger y Warrington, 1996), demostrando, en general, que las niñas muestran una mayor implicación e interés en la realización de los deberes escolares que los niños.

Por otro lado, no hay diferencias entre los tres cursos en el número de deberes realizados. Sin embargo, el cambio de segundo a tercer ciclo de Primaria parece que lleva consigo también un incremento en el tiempo dedicado a los deberes por parte de los alumnos. Por el contrario, el aprovechamiento del tiempo dedicado a los deberes va disminuyendo según se va avanzando de curso. Probablemente, el incremento del tiempo dedicado a los deberes de Segundo a Tercer Ciclo de Primaria no deba interpretarse en sí mismo como algo negativo, pero si el aprovechamiento del tiempo dedicado a los deberes va disminuyendo según se avanza de curso, entonces ya nos encontramos con dos cuestiones vinculadas con el tiempo dedicado a los deberes que deberán considerarse a la hora de explicar sus efectos sobre el rendimiento académico.

Los resultados de este trabajo permiten concluir que el rendimiento académico en los últimos cursos de Primaria (en las materias de Lengua Inglesa y Matemáticas) será mayor en la medida en que se realicen mayor número de deberes escolares de los prescritos, se aproveche mejor el tiempo utilizado para hacer los deberes y se dedique menos tiempo a la realización de esos deberes. Por tanto, según los resultados de este estudio, realizar un mayor número de deberes redunda en un mejor rendimiento académico, lo cual apoya la opinión de quienes defienden los deberes escolares como un instrumento útil y fundamental en la realidad escolar (Cooper et al., 2006; Epstein y Van Voorhis, 2001; Trautwein et al., 2006;). Además, es recomendable que esa mayor cantidad de deberes realizados, vaya acompañada de un mayor aprovechamiento del tiempo dedicado a los deberes y de menos tiempo invertido realizando esos deberes. 


\section{Referencias}

Becker, H. J. y Epstein, J. L. (1982). Parent involvement: A survey of teacher practices. Elementary School Journal, 83, 85-102.

Bouffard, T., Boileau, L. y Vezeau, C. (2001). Students' transition from elementary to high school and changes of the relationship between motivation and academic performance. European Journal of Psychology of Education, 16, 589-604.

Cohen, J. (1988). Statistical power analysis for the behavioral sciences ( $\left.2^{\mathrm{a}} \mathrm{ed}.\right)$. Hillsdale, NJ: Erlbaum.

Cooper, H. (1989). Synthesis of research on homework. Educational Leadership, 47(3), 85-91

Cooper, H. (2001). The battle over homework. Thousand Oaks, CA: Corwin Press.

Cooper, H., Jackson, K., Nye, B. y Lindsay, J. J. (2001). A model of homework's influence on the performance evaluations of elementary school students. Journal of Experimental Education, 69(2), 181-20.

Cooper, H. M., Lindsay, J. J., Nye, B. y Greathouse, S. (1998). Relationships among attitudes about homework, amount of homework assigned and completed, and student achievement. Journal of Educational Psychology, 9. $70-83$.

Cooper, H., Robinson J. C. y Patall, E. A. (2006). Does homework improve academic achievement? A synthesis of research, 1987-2003. Review of Educational Research, 76(1), 1-62.

Cooper, H. y Valentine, J. (2001). Using research to answer practical questions about homework. Educational Psychologist, 36(3), 143-153.

Cosden, M., Morrison, G., Albanese, A. y Macias, S. (2001). When homework is not homework: After-school programs for homework assistance. Educational Psychologist, 36(3), 211-221.

Covington, M. V. (1998). The will to learn: A guide for motivating young people. Cambridge, UK: Cambridge University Press.

De Jong, R., Westerhof, K. J. y Creemers, P. M. (2000). Homework and student math achievement in Junior High Schools. Educational Research and Evaluation, 6(2), 130-157.

Dettmers, S., Trautwein, U. y Lüdtke, O. (2009). The relationship between homework time and achievement is not universal: Evidence from multilevel analyses in 40 countries. School Effectiveness and School Improvement, 20, 375-405.

Deslandes, R. y Cloutier, R. (2002). Adolescents' perception of parental involvement in schooling. School Psychology International, 23(2), 220-232.

Epstein, J.L. y Van Voorhis, F.L. (2001). More Than Minutes: Teachers' Roles in Designing Homework. Educational Psychologist, 36(3), 181-193.

Finney S. J. y DiStefano C. (2006). Non-normal and categorical data in structural equation modelling. En G. R. Hancock, y R. O. Mueller (Eds.), Structural equation modelling. A second course (pp. 269-314). Greenwich, CT: Information Age Publishing.

Harris, S., Nixon, J. y Rudduck, J. (1993). School work, homework and gender. Gender and Education, 5(1), 3-14.

Hill, N. E. y Taylor, L. C. (2004). Parental school involvement and children's academic achievement: Pragmatics and issues. Current Directions in Psychological Science, 13, 161-164

Jackson, C. (2003). Motives for "laddishness" at school: Fear of failure and fear of the "feminine." British Educational Research Journal, 29(4), 583598.

Muhlenbruck, L., Cooper, H., Nye, B. y Lindsay, J. J. (2000). Homework and achievement: explaining the different strengths of relation at the elementary and secondary school levels. Social Psychology of Education, 3, 295317.

Núñez, J. C., Suárez, N., Cerezo, R., González-Pienda, J. A., Rosário, P., Mourão, R. y Valle, A. (2013). Homework and its relation to academic achievement across compulsory education. Educational Psychology. Doi: 1.1080/0144341.2013.817537.

Rosário, P., Mourão, R., Baldaque, M., Nunes, T., Núñez, J.C., GonzálezPienda, J.A., Cerezo, R. y Valle, A. (2009). Tareas para casa, autorregu- lación del aprendizaje y rendimiento en matemáticas. Revista de Psicodidáctica, 14(2), 179-192

Rosário, P., Mourão, R., Núñez, González-Pienda, J. y Valle, A. (2006). SRI and EFL homework: gender and grade effects. Academic Exchange Quarterly, 10(4), 135-14.

Rosário, P., Mourão, R., Núñez, J. C., y Solano, P. (2008). Homework and Self-Regulated Learning (SRL) at issue: Findings and future trends. En A. Valle, J. C. Núñez, R. G. Cabanach, J.A. González-Pienda y S. Rodríguez (Eds.), Handbook of instructional resources and their applications in the classroom (pp. 123-134). Nueva York: Nova Science Publishers.

Rosário, P., Mourão, R., Trigo, L., Suárez, N., Fernández, E. y TueroHerrero, E. (2011). Uso de diarios de tareas para casa en el inglés como lengua extranjera:evaluación de pros y contras en el aprendizaje autorregulado y rendimiento. Psicothema, 23(4), 681-687.

Ryan, E. M. y Deci, E. L. (2000). Self-determination theory and the facilitation of motivation, social development, and well-being. American Psychologist, 55, 68-78.

Senechal, M. y LeFevre, J. (2002). Parental involvement in the development of children's reading skill: A five-year longitudinal study. Child Development, 73, 445-46.

Vallerand, R. J., Fortier, M. F. y Guay, F. (1997). Self-determination and persistence in a real-life setting: Toward a motivational model of high school dropout. Journal of Personality and Social Psychology, 72, 1161-1176.

Trautwein, U. (2007). The homework-achievement relation reconsidered: Differentiating homework time, homework frecuency, and homework effort. Learning and Instruction, 17, 372-388.

Trautwein, U. y Köller, O. (2003). The relationship between homework and achievementFstill much of a mystery. Educational Psychology Review, 15, 115-145.

Trautwein, U., Köller, O., Schmitz, B. y Baumert, J. (2002). Do homework assignments enhance achievement? A multilevel analysis of 7 th grade mathematics. Contemporary Educational Psychology, 27, 26-5.

Trautwein, U., Ludtke, O., Schnyder, I. y Niggli, A. (2006). Predicting homework effort: Support for domain-specific, multilevel homework model. Journal of Educational Psychology, 98, 438-456.

Trautwein, U., Schnyder, I., Niggli, A., Neumann, M. y Lüdtke, O. (2009). Chameleon effects in homework research: The homework-achievement association depends on the measures used and the level of analysis chosen. Contemporary Educational Psychology 34, 77-88

Wagner, P., Schober, B. y Spiel, C. (2007). Time students spend working at home for school. Learning and Instruction, 18(4), 309-32.

Walker, J.M.T., Hoover-Dempsey, K.V., Whetselm, D. R. y Green, C. L. (2004). Parental involvement in homework: A review of current research and its implications for teacher, afterschool program staff, and parent leaders. Cambridge, MA: Harvard Family Research Project. Retrieved from http://www.gse.harvard.edu/hfrp/projects/fine/resources/homework. html

Younger, M. yWarrington, M. (1996). Differential achievement of girls and boys at GCSE: Some observations from the perspective of one school. British Journal of Sociology of Education, 17, 299-313.

$\mathrm{Xu}, \mathrm{J}$, (2006).Gender and homework management reported by high school students. Educational Psychology, 26, 73-91.

$\mathrm{Xu}, \mathrm{J}$. (2007). Middle school homework management: More than just gender and family involvement. Educational Psychology, 27, 173-189.

$\mathrm{Xu}$, J. (2010). Gender and homework management reported by African American Students. Educational Psychology, 3. 755-77.

Xu, J y Wu, H. (2013). Self-regulation of homework behavior: homework management at the secondary school level. Journal of Educational Research, 106(1), 1-13.

Zimmerman, B. J. y Kitsantas, A. (2005). Students' perceived responsibility and completion of homework: The role of self-regulatory beliefs and processes. Contemporary Educational Psychology, 3, 397-417. 